\title{
Research on influence of welding root node coupling on weld- ing deformation of large mining dump truck frame
}

\author{
Heping Xie ${ }^{1}$ \\ ${ }^{1}$ Xuzhou XCMG Mining Machinery Co. Ltd, Xuzhou, J S 221004, China
}

\begin{abstract}
Taking the frame of large-scale mining dump truck as the research object, it established the model of coupling or not of T-joint welding root by the finite element analysis, and analyzed the deformation of coupling or not of T-joint welding root. The deformation trend of the two cases is the same, which verifies the correctness of the prediction results of deformation. The results show that the maximum deformation occurs at the arc starting welding of the vertical plate, and the error between the simulation results and the test results is small. At the same time, the deformation error of gantry frame and hinge-hole at the key position can meet the production requirements, which is of great significance to the field production.
\end{abstract}

\section{Introduction}

Due to the characteristics of heavy load, large volume and bad working conditions, large-scale mining dump trucks have high requirements for structural design and process quality. The welding structure design combined with its special technical requirements has a direct impact on the following machining allowance, assembly process and service life. The welding structure design is reasonable $[1,2]$, which can effectively control the welding deformation and residual stress to maximize the carrying function of large mining dump truck. The welding structure design considers both the structure design and the welding process design, optimizes the structure design rationality and the welding process feasibility, which is the most typical application in the frame design of the large-scale mining dump truck. As the main loadbearing structure, the proportion of frame lightweight design, welding joint design and material selection in the design needs to be weighed. With the continuous optimization of frame structure form, the material tends to be conservative medium and low carbon steel with better welding ability, so the joint design is important. Much welding procedure qualification tests provide guarantee for joint performance, but in the welding process of large frame, the realization of welding procedure will be different according to the site environment temperature, fixture, blanking accuracy, welding quality, welding quality, welding assembly sequence, etc. To minimize the impact of production consistency on the frame welding quality, only relying on traditional on-site experience accumulation and other means cannot meet the cycle requirements of large-scale mining dump truck frame renewal; welding simulation technology has become an effective means.
At present, simulation can effectively predict the trend of deformation and residual stress. In prediction accuracy, the prediction accuracy is related to the equivalent conditions. On the basis of the inherent strain theory put forward by Japanese experts and scholars, Wang Yong [3] and others carried out simulation analysis on the welding deformation of the frame of the mining dump truck, and the error was within $20 \%$, which provided guidance for the frame production. In the equivalence of theoretical method and contact boundary condition, Fang Yuanbin et al. based on the thermo elasticplastic theory, equivalent the contact condition of largescale structure crane turntable fixture to the displacement constraint, and predicted the deformation, with the error controlled within $14 \%$, meeting the requirements of production and processing allowance. In improving the calculation efficiency, Zhao Haiyan of Tsinghua University [4] based on the local integral method, compared and analyzed the deformation of large thin-walled cylinder in different welding sequences, which was in good agreement with the test results, and the calculation time of the local integral method was only $1 / 6$ of that of the thermo elastic-plastic method. In improving the accuracy [5-7], the selection of heat source model, the form of spot welding, the type of element, the environment temperature, the parameters of heat radiation, the parameters of heat input and so on all affect the accuracy. In the actual production, the large-scale structural parts are limited by the measurement means, and there is almost no means to measure the overall size of the overall structural parts. Generally, the local key dimensions are measured, so the measurement results are affected by the local size effect, error accumulation and other factors. From the production point of view, the post welding structural parts are generally welded or orthopaedic, and then processed. The post welding structural parts need to ensure the size requirements of the key positions of the next process. 
Considering the relationship between production cycle and calculation efficiency, it is important to realize quantitative analysis of key positions of large-scale structural parts, which is directly related to the accuracy for largescale structural parts calculation.

For the large-scale mining dump frame, the typical joint root node coupling model is established, and the deformation analysis is carried out; similarly, based on the thermo elastic-plastic theory, the deformation is analyzed, and the deformation results measured by the experiment are verified.

\section{The establishment of finite element model}

\subsection{The process parameters.}

$\mathrm{T}$-joint is the typical joint form of the large-scale mining dump frame. The joint base plate size is $200 \times 100 \times$ $15 \mathrm{~mm}$, vertical plate size is $100 \times 100 \times 25 \mathrm{~mm}$. The groove is $60^{\circ}$, and the blunt edge is $2 \mathrm{~mm}$. The weld height is $30 \mathrm{~mm}$. The metal is Q690, which is welded by CO2 gas shielded welding and SLD70 solid-core welding wire. Process parameters are shown in Table 1.

Table 1. Process parameters welding of Q690.

\begin{tabular}{ccccc}
\hline & Arc voltage (V) & Arc current (A) & Velocity (mm/s) & Gas flow rate (L/min) \\
\cline { 2 - 5 } The left seam & $21-23$ & $210 \sim 220$ & 6 & 15 \\
The right seam & $26-28$ & $260 \sim 278$ & 7 & 20 \\
\hline
\end{tabular}

\subsection{Material parameters.}

Q690 is used for simulation, and some physical and mechanical parameters with temperature change are shown in Figure 1.

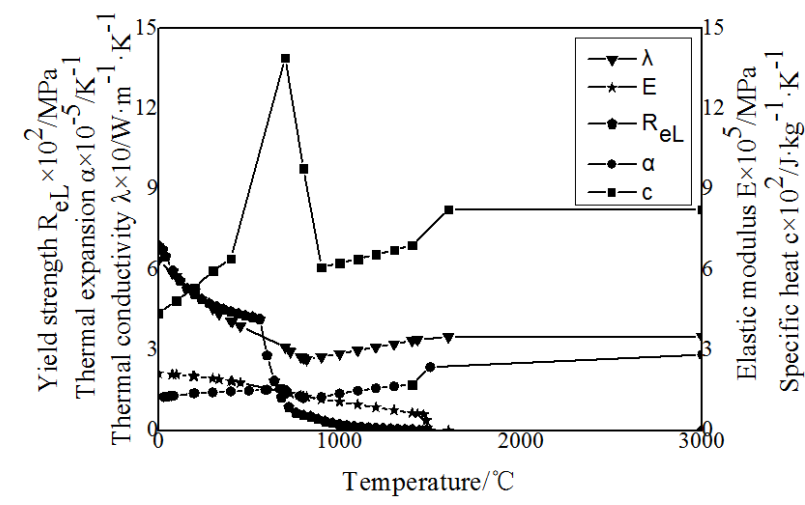

Figure.1 Relationship between thermo-mechanical parameters and temperature of Q690

\subsection{Mesh model.}

The model only calculates the deformation, considering the calculation time and manual mesh division time. The model mesh adopts single-layer mesh transition processing, the unit size of the weld position is $2 \mathrm{~mm}$, the total number of units is 21750 , and the number of nodes is 24861. According to the coupling or not, two kinds of models are established, as shown in Figure 2.

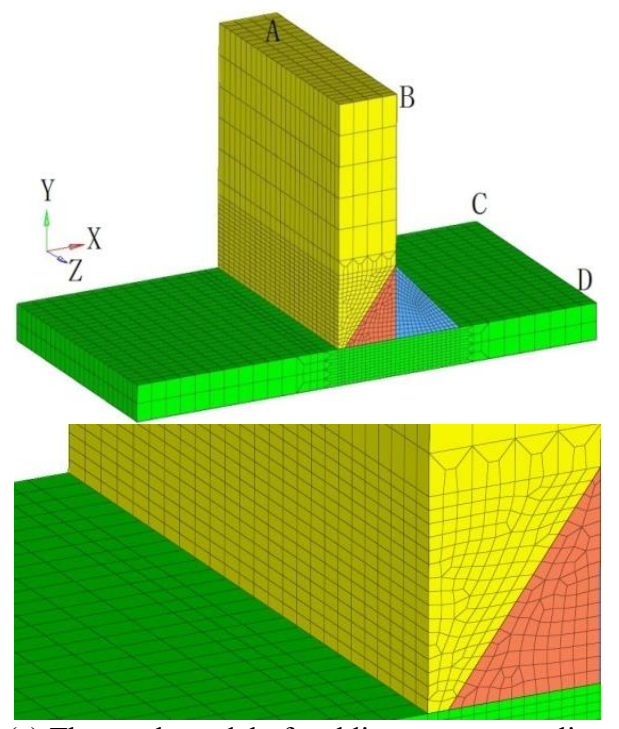

(a) The mesh model of welding root uncoupling

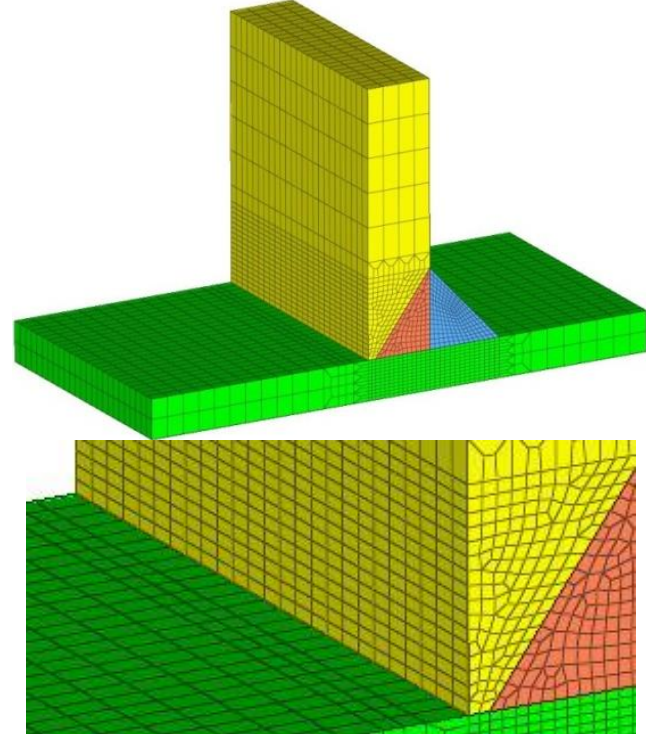

(b) The mesh model of welding root coupling

Figure 2. The finite element model 


\subsection{Material parameters.}

The double ellipsoid heat source model [8] is selected as the heat source model, which fully considers the characteristics of abrupt change of front temperature and slow change of rear temperature of carbon dioxide gas shielded welding heat source. The heat distribution functions of the front and back ellipsoids are in equation (1), (2).

$$
\begin{aligned}
& q(x, y, z, t)=\frac{6 \sqrt{3} f_{f} Q}{a_{1} b c \pi \sqrt{\pi}} e^{-3 x^{2} / a_{1}{ }^{2}} e^{-3 y^{2} / b^{2}} e^{-3 z^{2} / c^{2}} \\
& q(x, y, z, t)=\frac{6 \sqrt{3} f_{r} Q}{a_{2} b c \pi \sqrt{\pi}} e^{-3 x^{2} / a_{2}{ }^{2}} e^{-3 y^{2} / b^{2}} e^{-3 z^{2} / c^{2}}
\end{aligned}
$$

Where, $\mathrm{f}_{\mathrm{f}}$ and $\mathrm{f}_{\mathrm{r}}$ are heat flux distribution coefficients, $\mathrm{q}$ is input heat source power, $\mathrm{a}_{1}, \mathrm{a}_{2}, \mathrm{~b}$ and $\mathrm{c}$ are parameters defining ellipsoid shape, $a_{1}$, and $a_{2}$, respectively represent the length of front and rear ellipsoids, c affects weld width, and $b$ affects weld depth.

Because the typical joint weld bead adopts multilayer and multi-pass welding, the large model calculation of mining dump truck frame is carried out, not only the calculation time is long, but also the server configuration requirements are high, the calculation may consume the server floating point number, which is not suitable for production, and more exists in the laboratory research. The inherent strain is more suitable for qualitative calculation, and the calculation results are greatly affected by boundary conditions; the calculation results of similarity theory and local integral method are affected by poor material consistency and size effect in production, so it is difficult to promote them in large-scale structural parts of complex structures. The steady-state heat source is used to load; the multi-layer and multi-pass welding is simplified into two-layer welding, one layer of groove and one layer of cover welding.

\subsection{Mechanical boundary conditions.}

The mechanical boundary conditions are constrained by displacement and spring, and two joint positions are selected on the lower surface of the middle section in the width direction to limit the $\mathrm{x}$ displacement without affecting the longitudinal shrinkage deformation. The weld back is set the node along the length of the weld seam limits the z-direction displacement, which does not affect the transverse contraction. To avoid the insufficient constraint caused by the setting of single direction displacement constraint and the excessive constraint caused by the two-way displacement constraint, the y spring constraint is set on the surface of the element selected at the back center of the weld seam.

\section{Analysis of typical joint calculation results}

\subsection{Analysis and verification of vertical plate welding deformation.}

Through the simulation calculation, the deformation of T-joint root node coupling or not is obtained respectively. The arc starting welding is located at the end of point $\mathrm{A}$ and point $\mathrm{C}$, and the $\mathrm{x}$-direction deformation results of vertical plate root node coupling or not are extracted respectively. At the same time, obtain the joint sample through the test, and measure the deformation at the positions $0,50 \mathrm{~mm}, 100 \mathrm{~mm}, 150 \mathrm{~mm}$ and $200 \mathrm{~mm}$ from point $\mathrm{B}$ respectively with feeler gauge to get the deformation curve, as shown in Figure 3.

From Figure 3 that the peak value of welding root coupling X-direction deformation is $0.76 \mathrm{~mm}$, the peak value of root node uncoupling deformation is $0.94 \mathrm{~mm}$, and the maximum deformation position occurs at the arc starting position of vertical plate. The deformation trend is the same in both cases, which is consistent with the deformation law of T-joint [6], which verifies the correctness of the prediction results.

The maximum error of welding root node uncoupling between the calculation results and the test results is $9.7 \%$, and the maximum error of welding root node coupling is $28.6 \%$. The larger error is related to the smaller deformation. From deformation trend, the $\mathrm{x}$-direction deformation along the BA direction decreases gradually, and the degree of deformation decrease fluctuates to a certain extent, which may be related to the uniformity of mesh division. The deformation of starting position $a$ is small, and the welding gap of ending position B may be gradually increased due to the welding of starting first, and the deformation of ending position will be increased due to the cooling contraction of welding first.

\subsection{Analysis and verification of bottom plate welding deformation.}

Similarly, the simulation and test results of deformation in $\mathrm{Y}$ direction are extracted from point $\mathrm{D}$ as $0,50 \mathrm{~mm}$, $100 \mathrm{~mm}, 150 \mathrm{~mm}, 200 \mathrm{~mm}$, as shown in Figure 4 .

From figure 4, the peak value of coupling deformation in Y direction of T-joint root node is $2.43 \mathrm{~mm}$, the peak value of uncoupling deformation is $2.56 \mathrm{~mm}$, and the maximum deformation are all at the arc starting position of bottom plate. The maximum error is $3.2 \%$ and $4.9 \%$ respectively. From the deformation trend, the $\mathrm{x}$-direction deformation along the DC direction decreases gradually, and the degree of deformation decrease fluctuates to a certain extent. The deformation of arc starting position $\mathrm{C}$ is small.

In conclusion, the error of welding root node uncoupling is small. Therefore, in the simulation analysis of large-scale structure, it is suggested that the T-joint should be treated in the way of welding root node uncoupling. 


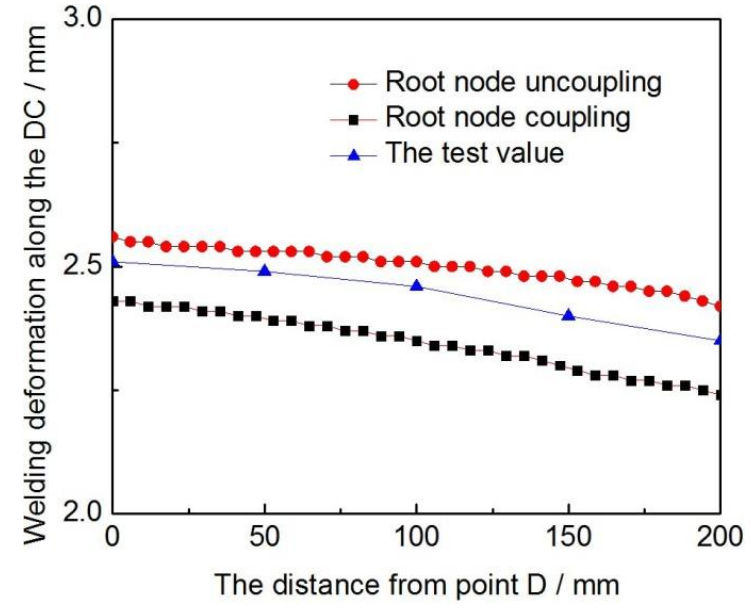

Figure 3. Deformation of X-direction

\section{Analysis of frame calculation results}

\subsection{Establishment of frame finite element mod- el.}

In above analysis of the influence of deformation trend fluctuation and the total number of grids on the calculation efficiency, grid transition processing is adopted, with the weld position unit size of $2 \mathrm{~mm}$, the number of grids of 2271937 and the number of nodes of 2577010 . The welding root node is uncoupled, and the frame model is established, as shown in Figure 5.

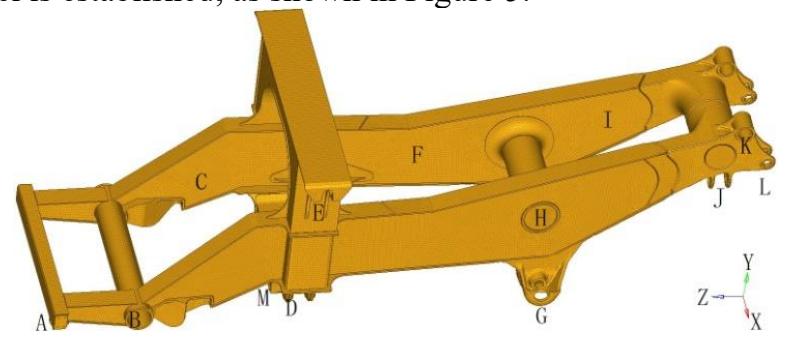

Figure 5. The finite element model of frame

The process parameters, heat source model and material parameters of the frame model are set to the above typical joint parameters. To ensure the structural rigidity of the frame and to take into account the fixture used on site, the mechanical boundary conditions are constrained by displacement and spring. To simulate the supporting effect of the platform on the frame, spring restraint is used on the lower surface of point $\mathrm{A}$ and point $\mathrm{M}$ of the frame; To simulate the supporting effect of the fixture of the hinge hole, $\mathrm{Z}$ and $\mathrm{Y}$ restraint are set at point $\mathrm{B}$, point $\mathrm{G}$, point $\mathrm{H}$, point $\mathrm{K}$ and point $\mathrm{L}, \mathrm{X}$ and $\mathrm{Y}$ restraint are set at point $\mathrm{D}$ and point $\mathrm{J}$; To simulate the restraining effect

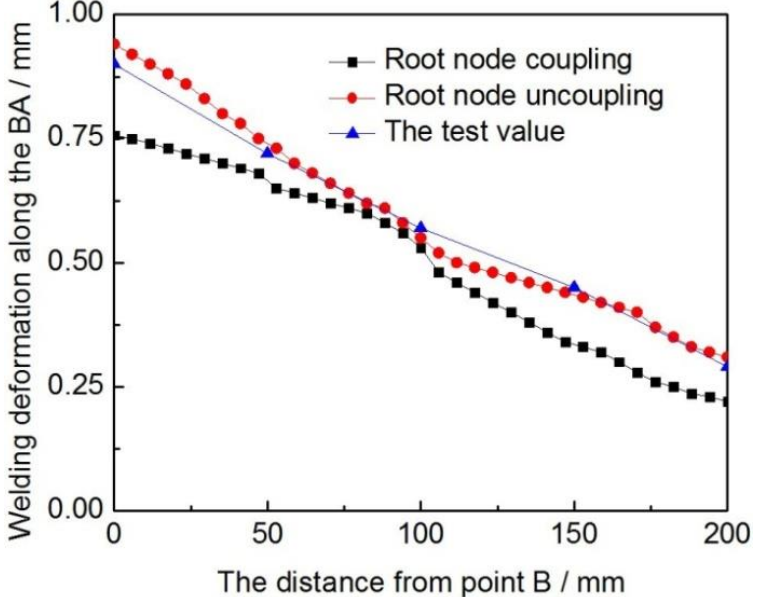

Figure 4. Deformation of Y-direction

of the brace and fixture on the model, $\mathrm{X}$ restraint is set at point $\mathrm{C}$, point $\mathrm{E}$, point $\mathrm{F}$ and point $\mathrm{I}$.

\subsection{Analysis and verification of the key position welding deformation.}

As the gantry frame point $\mathrm{E}$ is corrected and the hinge hole points $\mathrm{G}, \mathrm{K}$ and $\mathrm{l}$ in the next process are machined, the deformation of these points is focused. Considering that the casting design is adopted for the parts at point $\mathrm{K}$ and point 1 at the rear of the frame, the shrinkage deformation is small, so the deformation at point $\mathrm{G}$ and gantry frame point $\mathrm{E}$ is not considered. The deformation results of the frame are obtained, and the single side deformation results of the key positions of the frame are extracted. The deformation of E point is $6.4 \mathrm{~mm}$, and that of $\mathrm{G}$ point is $5.4 \mathrm{~mm}$.

As the requirements of frame process design on site, the same batch of frame shall be sampled for measurement. The measurement tools shall be the special threedimensional platform on site, and the deformation before and after welding of point $\mathrm{E}$ and point $\mathrm{G}$ shall be recorded, 3 groups in total, as shown in Table 2 .

From Figure 4(b), the long plate has wavy deformation, and the curve of the bottom pad at the point 4-6 is consistent with the trend of the strip deformation. The measured 4 and 5 points are in good agreement with the simulation results. The measured point 6 has $0.27 \mathrm{~mm}$ deviation from the simulation results. At the same time, the peak position of the measured point and the simulation curve peak is similar. There are 5 peaks in the deformation of the long strip, and the deformation ranges from $0.08 \mathrm{~mm}$ to $0.36 \mathrm{~mm}$. The wave deformation of the long strip is mainly related to the weld seams, such as the bottom pad, the vibrator, the long strip and the front $\mathrm{Z}$ plate. 
Table 2. Deformation measurement before and after frame welding ( $\mathrm{mm})$.

\begin{tabular}{lccccccc}
\hline & before welding & after welding & Difference & & before welding & after welding & Difference \\
\cline { 2 - 7 } $\mathrm{E}_{1}$ & 3162.5 & 3152.9 & 9.6 & $\mathrm{G}_{1}$ & 3073.5 & 3065.1 & 8.4 \\
$\mathrm{E}_{2}$ & 3164 & 3153.3 & 10.7 & $\mathrm{G}_{2}$ & 3075 & 3065.8 & 9.2 \\
$\mathrm{E}_{3}$ & 3163.5 & 3152.4 & 11.1 & $\mathrm{G}_{3}$ & 3074.5 & 3063.7 & 10.8 \\
\hline
\end{tabular}

Through the calculation of the average value, the deformation of point $\mathrm{E}$ is $10.5 \mathrm{~mm}$, and that of point $\mathrm{G}$ is $9.5 \mathrm{~mm}$. The error of point $\mathrm{E}$ is $18.0 \%$, and that of point $\mathrm{G}$ is $12.0 \%$. For the production of large-scale mining dump truck frame, the length dimension of the frame in the longitudinal symmetrical plane direction is up to 12 meters, the deformation of the key position is about 10 $\mathrm{mm}$, and the error is controlled within $18 \%$, which can meet the production requirements. Considering various control methods to reduce the deformation, it is inevitable to increase the residual stress of the structure, and the deformation of about $10 \mathrm{~mm}$ can be one the residual stress of the structure can be relieved to a certain extent, and the deformation can be solved by means of repair welding and orthopedic to meet the production requirements.

\section{Conclusions}

A finite element model is established to analyze the deformation of the frame of a large-scale mining dump truck by selecting the typical joint form at the key position and establishing the coupling or not of the T-joint welding root. At the same time, it is widely used in the frame of large-scale mining dump truck. Through the research, the following conclusions are drawn: whether the T-joint root node is coupled or not, the maximum deformation position occurs at the arc starting position, the deformation trend is the same in both cases, which verifies the correctness of the prediction results of welding deformation. The error between the simulation results of the welding root node uncoupling and the test results is small. It is suggested that the large-scale mining dump truck frame adopt the treatment of welding root node uncoupling. The deformation error at the position of gantry frame and hinge-hole meets the production requirements. The welding deformation is only studied, and the residual stress will be released continuously in engineering application, which needs further analysis and research.

\section{References}

1. Tian X T: Welding Structure (China Machine Press Publications, China 1982).

2. Peric M, Tonkovic Z, Rodic A, Surjak M, Garašic I, Boras I and Švaic S 2014 Mater. Design 53 10521063.

3. Wang Y, Ge C, and Xiu X T 2017 Min.Equip. (2) 54-55.
4. Zhao H. Y., Wu J W, Lu X M, Jian B, and Li H W 2017 J. Tsinghua Univ (Sci. \& Tech.). (5) 17-21.

5. Deng D, Liu X, He J and Liang W 2016 Int.J. Adv. Manuf. Tech.82(5-8) 1049-1062.

6. Wang J C, Ma N, Murakawa H, Teng B and Yuan S 2011 Mater. Design32 4728-4737.

7. Rong Y M, Chang Y, Xu J Y, Huang Y, Lei T and Wang C 2017Int. J. Pres. Ves. Pip.15851-58.

8. Fang Y B, Zong X M, Xiao Y, Liang J, and Yin X Q 2016 Elec. Weld. Mach. 46(8) 17-21. 